\title{
The Silencing Face of DNA Replication: Gene Repression Mediated by DNA Replication Factors
}

\author{
Patricia Chisamore-Robert, Daniel Jeffery and Krassimir Yankulov \\ Department of Molecular and Cellular Biology, University of Guelph \\ Canada
}

\section{Introduction}

DNA replication in eukaryotes initiates at multiple origins. The activation of these origins is a critically important event in the life of each cell and is tightly regulated by numerous highly conserved trans-factors.

Saccharomyces cerevisiae origins (called Autonomously Replicating Sequences, ARSs) contain a core $A$ element called ACS ( $\underline{A} R S$ Consensus Sequence), plus an array of auxiliary $B$ elements. Most ARSs fire at their chromosomal positions, but there are numerous dormant $A R S$ s as well. Instead of being origins, these dormant $A R S s$ serve as silencer elements, which function in the epigenetic repression of nearby genes. Even more, many DNA replication trans-factors have also been reported to affect gene silencing. This puzzling functional duality of ARS and DNA replication factors has attracted significant interest. Evidence from other species has suggested that the overlap between gene silencing and DNA replication operates in other eukaryotes. In this chapter we will review in detail the activity of ARSs as origins of replication and as silencers. We will focus on sequence dissimilarities between silencer and origin $A R S s$ and will propose a model for the functional duality of DNA replication factors.

\subsection{Origins of DNA replication in S.cerevisiae}

Eukaryotic origins of DNA replication display a significant inter-species diversity. In higher eukaryotes this diversity reaches a point where origin locations are difficult to identify by homology search (Mechali, 2010). A remarkable exception of this diversity occurs in the yeast S.cerevisiae. In this organism the first functional origins have been identified by screens for DNA elements which confer DNA replication on plasmids (Stinchcomb et al., 1979; Chan \& Tye, 1980; Kearsey, 1983). Comparison between these autonomously replicating sequences (ARSs) have shown that they encompass approximately 200bp of DNA and contain perfect or one-base mismatches to the $11 \mathrm{bp}$ ARS consensus sequence (ACS) $5^{\prime}$ WTTAYRTTTW-3' (where $\mathrm{W}=\mathrm{A} / \mathrm{T} ; \mathrm{Y}=\mathrm{C} / \mathrm{T} ; \mathrm{R}=\mathrm{A} / \mathrm{G}$ ). Linker scanning substitutions in several ARSs have determined that the ACS is the sole essential element for DNA replication (Marahrens \& Stillman, 1992; Rao et al., 1994; Lin \& Kowalski, 1997; Chang et al., 2008). However, auxiliary $B$ elements (B1-B4) within $A R S$ s are also necessary for full origin activity (Marahrens \& Stillman, 1992; Lin \& Kowalski, 1997). The B2, B3 and B4 elements are not 
present in all ARSs. B2 is a site for the unwinding of DNA while B3 is a binding site for Abf1p (ARS-binding factor 1), which is a protein involved in numerous chromatinassociated functions including DNA replication, gene silencing, transcriptional activation and DNA repair (Rehman \& Yankulov, 2009). The function of B4 is unknown, but its mutation reduces replicator activity (Lin \& Kowalski, 1997). Interestingly, the destruction of more than one of the $B$ elements substantially reduces origin firing activity (Marahrens \& Stillman, 1992) and ACS alone is not sufficient to confer replicator activity at natural yeast chromosomes (Raghuraman et al., 2001).

The $B 1$ element, along with the ACS, is found in all known $A R S$ s and forms a bipartite binding site for the Origin Recognition Complex (ORC) (Rao \& Stillman, 1995). However, the $B 1$ sequence is not nearly as conserved as ACS. The cross- $A R S$ homology at the putative position of $B 1$ has been identified as a WTW motif found 17-19 bp upstream of the ACS (Chang et al., 2008) or an AWnY (W=A/T; $\mathrm{Y}=\mathrm{C} / \mathrm{T} ; \mathrm{n}=$ any nucleotide) motif 16 bases upstream of ACS (Palacios DeBeer et al., 2003). Even more, the whole region upstream of ACS is A/T rich thus providing multiple nearby WTW/AWnY motifs. Ultimately, the precise position of $B 1$ and its significance remains somewhat elusive.

\subsection{ARSs initiate replication}

A wealth of information has been accumulated on the mechanisms by which ARSs initiate DNA replication (Fig. 1). ORC, which is built of six different Orc proteins, binds the ACS-B1 elements to nucleate the formation of the pre-replicative complexes (Blow \& Dutta, 2005; Labib, 2010). Shortly after mitosis, Cdt1p and Cdc6p recruit the heterohexameric MCM complex to the ARS-bound ORC. Thus, ARSs are poised to initiate DNA replication upon receiving a regulatory stimulus. This stimulus is provided in S-phase by two protein kinases, DDK and CDK2 (Labib, 2010). It seems that the critical event in the stimulation of origins is the phosphorylation of Mcm4p (Sheu \& Stillman, 2010) by DDK. However, other components of the pre-initiation complex are also phosphorylated with similar timing (Labib, 2010). These events culminate in the activation of the MCM helicase, in the unwinding of origin DNA and in the assembly of the DNA replication machinery.

There are about 12000 matches or near-matches to ACS in the genome of S. cerevisiae (Nieduszynski et al., 2006). Of these, only 500-700 are loaded with ORC and MCM proteins (Wyrick et al., 2001) and only about 400 initiate DNA replication (Raghuraman et al., 2001). In general, the early firing origins are located in the central portion of the chromosomes, while the later firing origins are found at the periphery (Raghuraman et al., 2001). It is believed that the same initiation events take place at all origins of DNA replication, but at different times throughout S-phase. Interestingly, the dormant origins positioned in the immediate subtelomeric regions or at the mating type loci (see below) also recruit the ORC and the MCM complexes (Wyrick et al., 2001; Rehman et al., 2006), but seldom if at all fire. It is not known how DDK, CDK2 and other initiation factors are regulated to confer the temporal pattern of origin firing, how they discriminate dormant origins or how all these events are coordinated.

The disparity between loading and firing of ARSs in S.cerevisiae is reminiscent to the situation in metazoans, where tens of thousands of genomic positions are primed as origins, but only a small subset actually fire. It is believed that this excess of available origins can accommodate the significant differences in growth conditions during metazoan development as well as the substantial variation in chromatin structure in different cell 
types (Mechali, 2010). For example, local chromatin structure, transcription and/or different environmental and physiological conditions will contribute to the selection of the most suitable origins. In this way, unnecessary interference with gene expression or the disturbance of established heterochromatin domains will be avoided.

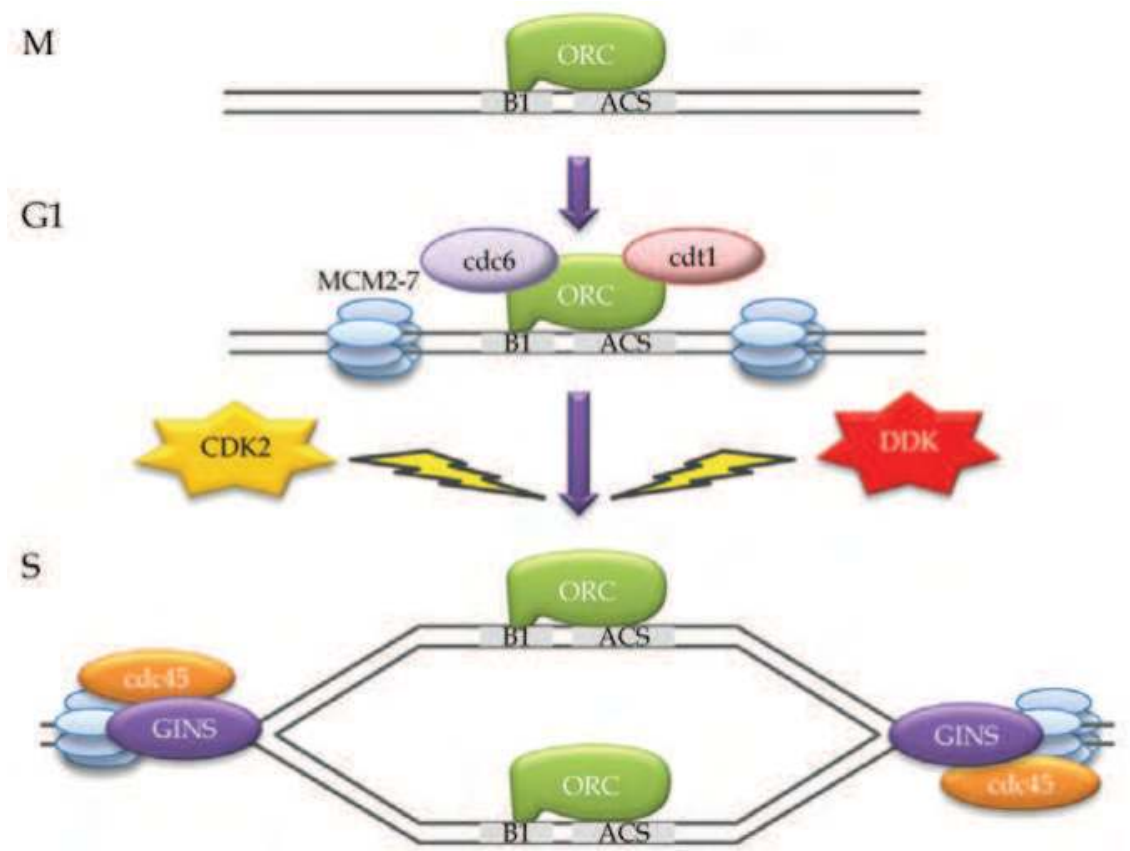

Fig. 1. Origin activation in S.cerevisiae - ORC binds the ACS-B1 elements. In early G1 phase, ORC recruits Cdc6p and Cdt1p. In turn, Cdc6p and Cdt1p load the hexameric helicase complex MCM2-7. In the G1/S transition, the Dbf4-dependent kinase DDK (also known as Cdc7p) and the Clb5-dependent kinase CDK2 (Cdc28p) phosphorylate the MCM2-7 complexes to trigger their helicase activity. DDK also phosphorylates Cdc45p, which is then able to recruit the GINS and other elongation factors for the progression of DNA replication.

The so-called Jesuit model ("For many are called, but few are chosen" (Matthew 22:14, the Bible)) has been proposed to explain the limited firing of origins. This model implicates that the considerable flexibility of DNA replication programs is most likely controlled by the abundance of pre-replicative complex factors (such as ORC and MCM2-7) and a corresponding limitation of initiation factors (such as Cdc45, Cdc7p and CDK2) (DePamphilis, 1993). Budding yeast provides an interesting twist to this model. Not only are certain origins chosen to fire while others are not, but some of the non-firing origins aqcuire a completely new role and contribute to the local silencing of genes.

\subsection{ARSs act as silencers}

Eukaryotic genes are regulated by a variety of mechanisms including complete silencing via condensed heterochromatin structure. The condensed/relaxed chromatin structures are 
faithfully transmitted to daughter cells thus ensuring the continuity of gene expression programs. This intriguing epigenetic phenomenon has been extensively studied at the mating type (HMRa and $H M L \alpha)$ (Fig. 2) and at the telomeric loci (Fig. 3) of S.cerevisiae. At all these loci the critical role in gene silencing is played by the SIR (Silent Information Regulator) proteins (Rusche et al., 2003). Through contacts with DNA-binding proteins, Sir1p, Sir3p and Sir4p recruit the Histone-Deacetylase Sir2p. In turn, Sir2p deacetylates the tails of $\mathrm{H} 3 / \mathrm{H} 4$ histones on the nearby nucleosome. Additional Sir3p and Sir4p then associate with the deacetylated histone tails to recruit more Sir $2 \mathrm{p}$ and expand the domain of deacetylated nucleosomes. Ultimately, the deacetylation of histones culminates in the establishment of compacted heterochromatin, which suppresses gene expression. The spreading of SIR proteins is countered by Histone-Acetyl-Transferases and other factors, whose identity and modes of action are not so well understood (Lafon et al., 2007; Ehrentraut et al., 2010).

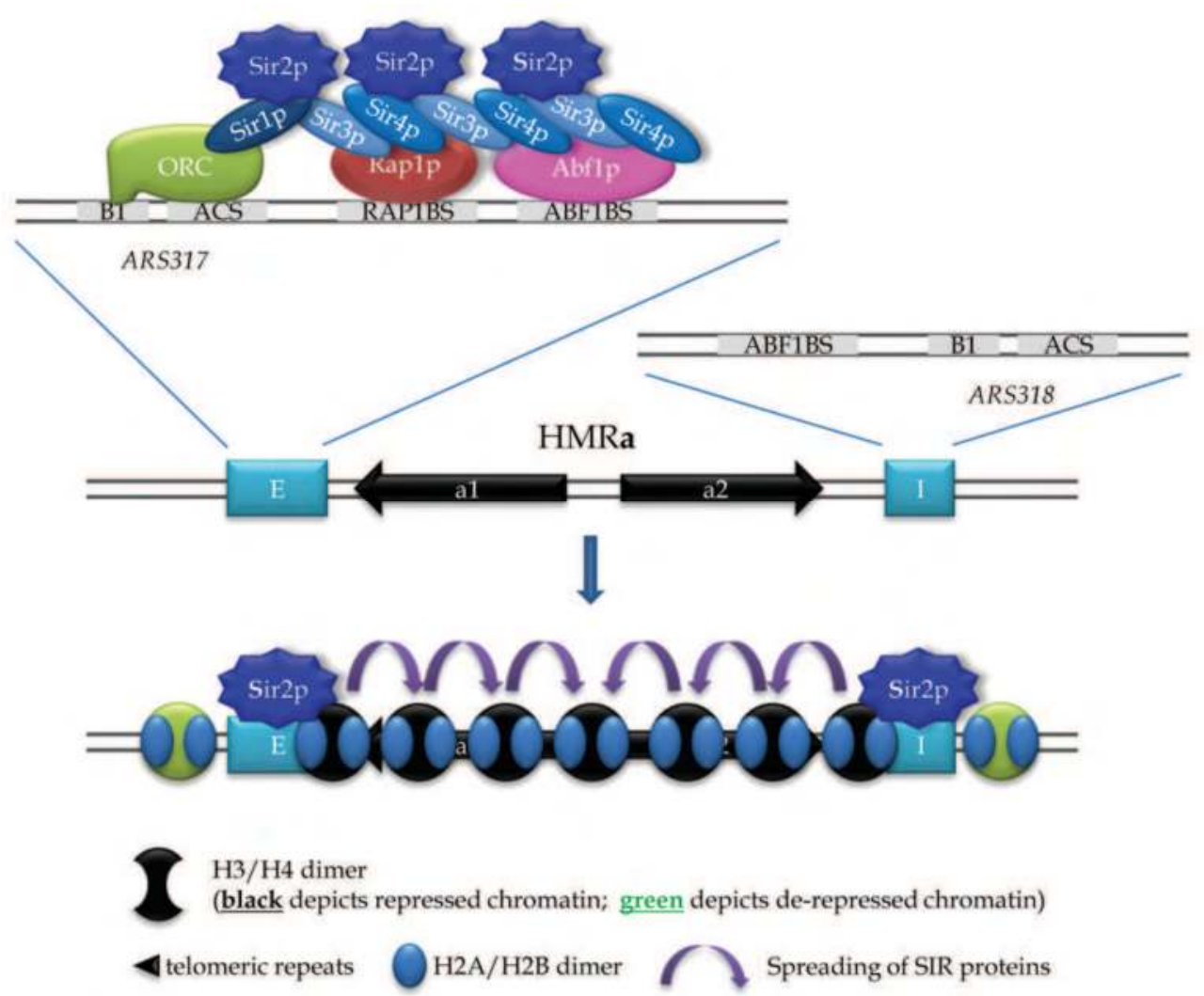

Fig. 2. Gene silencing at the HMRa locus - ARS317 and ARS318 recruit ORC (only recruitment by ARS317 is shown), which in turn recruits Sir1p. Both Rap1p and Abf1p recruit Sir3p and Sir4p. The tethering of Sir1,3,4p confers the nucleation of the silenced domain and recruits Sir2p. Sir2p deacetylates adjacent histone tails, which recruit more Sir3p/Sir4p and contribute to the spreading of SIR proteins, as demonstrated (blue arrow). 
The silent mating type loci, $H M R \mathbf{a}$ and $H M L \alpha$, are constitutively and completely repressed by robust heterochromatin structure. The genes encoded by these loci are expressed only when translocated to the MAT locus (Rusche et al., 2003). In turn, the MAT locus can accomodate and express only the a or the $\alpha$ genes. In this way, it is gauranteed that no erraneous expression of the two opposing mating types occurs.

HMRa and HML $\alpha$ are each flanked by $E$ and I silencers (Fig. 2). Remarkably, ARSs have been identified as essential elements in all four silencers of the these loci (Abraham et al., 1983; Broach et al., 1983; Rusche et al., 2003). For example, the HMRa-E silencer contains ARS317 as well as binding sites for Rap1p and Abf1p, whereas the HMRa-I silencer contains ARS318 and an Abf1p binding site (Fig. 2). Depending on the genomic context, both Abf1p and Rap1p bind to gene silencers or activator elements (Shore \& Nasmyth, 1987; Shore et al., 1987). Just as in replication origins, ORC binds to the bipartite ACS-B1 of the ARSs in the mating type loci silencers. However, instead of recruiting replication machinery, the Orc1p subunit of ORC recruits Sir1p, while Rap1p and Abf1p bind and recruit Sir3p and Sir4p. As shown in Fig. 2, Sir1p, Sir3p and Sir4p recruit Sir2p to establish a focal point of silencing and initiate the spreading of the SIR proteins. Similar events take place at ARS318 in the HMRa-I silencer.

It is important to note that the ARSs of the mating type loci are not substantially different from replicator ARSs. Both types of $A R S$ bind to ORC in vivo and in vitro (Palacios DeBeer et al., 2003). If placed on a plasmid, the silencer $A R S$ s act as perfectly good origins of DNA replication (Chan \& Tye, 1980). The opposite is also true; replicator ARSs can acquire silencer activity when inserted in the mating type loci (McNally \& Rine, 1991; Weinreich et al., 2004; Casey et al., 2008).

\subsection{ARSs act as proto-silencers}

ARSs also play a somewhat similar silencing role at the telomeres (Fig. 3). At these loci, the telomeric repeats act as the principal silencers while $A R S s$ have a silencer-enhancing role (Fourel et al., 2002). The telomeric $\mathrm{TG}_{1-3}$ repeats provide multiple binding sites for Rap1p. Similar to the mating type loci, Rap1p recruits Sir3p and Sir4p to establish the initiation point for the SIR protein spreading (Fourel et al., 2002; Rusche et al., 2003). ARSs and Sir1p are not required for this step. However, the absence of subtelomeric ARSs or Sir1p significantly reduces the span of the silenced domain and its stability while the artificial tethering of Sir1p to the telomere boosts the silencing of nearby genes (Chien et al., 1993). Thus, subtelomeric ARSs and their ability to recruit Sir1p through Orc1p play an important, yet secondary role in gene silencing at the telomeres. At other locations, isolated ARSs do not induce gene repression, but can boost the activity of an existing silencer. For this reason they were classified as proto-silencers (Fourel et al., 2002).

The complexity of telomeric silencing does not end there. Besides ARSs, the repetitive Core $X$ and $Y^{\prime}$ elements in the sub-telomere also contain isolated Rap1p and Abf1p binding sites. All these act as weak multiple proto-silencers. In addition, the Core $X$ and $Y^{\prime}$ elements harbour anti-silencer modules called sub-telomeric anti-silencing regions (STARs) (Fourel et al., 1999; Fourel et al., 2004; Power, 2011). The combined assembly of proto-silencers and weak anti-silencers produces a multitude of variations in the strength, stability and spreading of telomeric silencing (Fourel et al., 2004). Even more, Core $X$ and $Y^{\prime}$ elements contain isolated clusters of telomeric $\mathrm{TG}_{1-3}$ repeats and are able to interact with the telomeres forming t-loop and D-loop structures. The folding back of telomeric DNA brings the SIR 


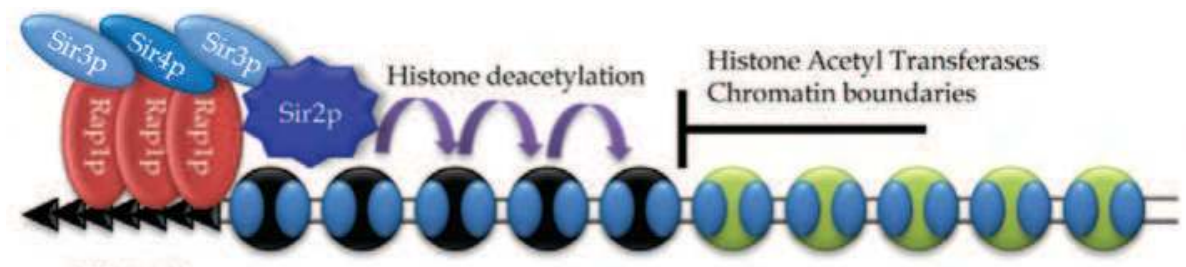

Telomere
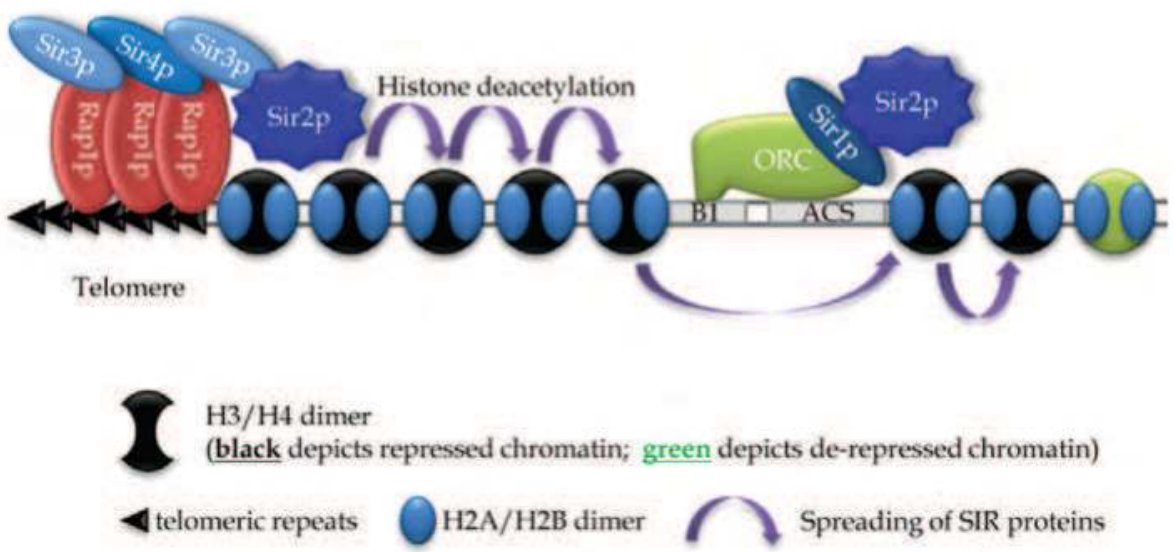

Fig. 3. Gene silencing at the telomeres - Rap1p binds to the telomere and recruits Sir3p and Sir4p. The recruitment of Sir $2 p$ and the spreading of SIR proteins is as explained in the text. Subtelomeric ARSs recruit ORC and Sir1p and enhance the spreading of SIR proteins and histone deacetylation away from the telomeres. This spreading is countered by HistoneAcetyl-Transferases and is limited by chromatin boundaries and insulators. Repressed chromatin acts to silence any genes wrapped within it while genes within de-repressed chromatin remain active.

proteins bound to the telomeric repeats into close proximity with those bound to the subtelomeric Core $X$ element. This interaction creates a highly condensed heterochromatic structure in a specific region of the sub-telomere while the stretch of DNA between the Core $X$ and the telomere may actually be euchromatic. The formation of these fold-back structures generates discontinuous telomeric silencing and strong silencing domains can be formed many kilobases away from the telomere (Pryde \& Louis, 1999; Fourel et al., 2004). Importantly, as any other ARSs, telomeric proto-silencer ARSs also contain a normal ACS-B1 module, bind ORC and act as origins when placed on mini-chromosomes (Wyrick et al., 2001; Rusche et al., 2003; Chan \& Tye, 1980).

\section{Results}

\subsection{What determines the activity of $A R S s ?$}

As mentioned earlier, replicator and silencer $A R S s$ are almost completely interchangeable. For example, $A R S$ s derived from origins can recapture the silencer activity in HMRa and the 
proto-silencer activity at the telomere when transferred to these positions (Palacios DeBeer et al., 2003; Weinreich et al., 2004; Casey et al., 2008; Rehman et al., 2009). Telomeric and silencer ARSs also act as replicators when moved to a plasmid (Chan \& Tye, 1983). Even more, many bona fide DNA replication factors have also been identified as silencing factors and mutations in them affect both the efficiency of origins and the epigenetic silencing at telomeres and the mating type loci (Axelrod \& Rine, 1991; Ehrenhofer-Murray et al., 1999; Rehman et al., 2006).

So, what confers the functional plasticity of ARSs? The fair answer is that we do not really know. Many studies have correlated the efficiency of origins to their proximity to heterochromatin (Weinreich et al., 2004; Field et al., 2008; Mechali, 2010). Indeed, origins in compact chromatin tend to fire less frequently than origins in open chromatin. It is conceivable that open chromatin is necessary for the assembly of the pre-replicative complexes (Doyon et al., 2006; Espinosa et al., 2010). However, chromatin structure is not the only regulator of origin activity. For example, in the mini-chromosome maintenance assay, (which involves the transfer of different origins to a plasmid and examining their efficiency under the same genetic context), it was found that different $A R S$ s fire at different rates and support different levels of DNA replication (Chan \& Tye, 1980; Chang et al., 2008). These observations immediately suggest that variation in the sequence of ARSs must also play a role in the fine tuning of ARS function.

The next step was to take highly efficient $A R S$ s from euchromatic regions and insert them into heterochromatic regions known to have poor replication initiation efficiency. Consequently, the high efficiency of the ARSs was lost, showing that the genomic context was powerful enough to overcome the effects of the sequence variation (Weinreich et al., 2004). However, a parallel change in the activity of ARSs has been discovered. Not only were the relocated $A R S$ s showing reduced replication and late firing, they were now boosting the epigenetic silencing of the nearby genes, contrary to their activities in their native chromosomal locations. A fine twist to these phenomena is that the replicator ARSs did not make perfect silencers (Casey et al., 2008) or proto-silencers (Rehman et al., 2009), reflecting the fact that silencer $A R S$ s sometimes do not make perfect replicators (Chang et al., 2008; Palacios DeBeer et al., 2003). It is not clear how the new chromatin environment of the relocated ARSs has contributed to their functional conversions.

This leads us to the question: is there any feature of an ARS that determines its predisposition to act as a silencer or a replicator? We and others have recently aligned a number of $A R S s$ in search for some correlation between sequence and function. It was possible to delineate a $B 1$ element consensus (WTW) from the origins on chromosome III (Chang et al., 2008). However, silencer and telomeric ARSs showed even lower conservation of this B1 element compared to other ARSs (Rehman \& Yankulov, 2009). Another line of evidence has previously shown that $B 1$ can modulate the affinity of ACS-B1 to ORC in vitro and that silencer ACS-B1 have higher affinity to ORC (Palacios DeBeer et al., 2003).

We have hypothesised that the mode of ORC association to ACS-B1 can ultimately influence how well an ARS will act as an origin or a silencer. If this is the case, variations in the B1 element and its flanking sequences can potentially contribute to the functional conversions of ARSs. For example, if $B 1$ causes ORC to acquire a specific conformation and higher affinity, ORC could end up recruiting the silencing machinery through the Orc1p-Sir1p interaction. In addition, it is also possible that the orientation of ACS-B1 towards another silencer could have an impact on the conformation of ORC, again promoting epigenetic 
silencing rather than replicator function. In both situations, ACS-B1 should provide for a significant level of flexibility of ORC, which in turn should allow the acquisition of silencer/replicator function depending on the chromatin context. In the following sections we will present our on-going studies that are testing these models.

\subsection{Destruction of $B 1$ has different effects in silencer and replicator $A R S s$}

Initial assessment of the role of the B1 element has been performed on two well characterised ARSs, the replicator ARS1 and the telomeric proto-silencer ARS319 (Fig. 4). We inserted these origins with an adjacent $U R A 3$ reporter in the left telomere of chromosome VII and assessed the level of repression by a routine assay for the sensitivity of cells to FOA (5-Fluoro-Orotic Acid). FOA is a neutral substance, which is turned into a toxin by the URA3-encoded Orotidine-5'-phosphate-decarboxylase. Hence, cells with repressed URA3 will grow in the presence of FOA, while cells expressing URA3 will be sensitive to FOA. After transforming with the integrating constructs, cells were selected on media without uracil (SC-ura) and telomeric integration was confirmed by PCR. The transformed cells were then grown in non-selective media for 15-20 generations to reach equilibrium of epigenetically repressed and transcribed URA3 and then plated on non-selective plates and plates containing FOA. The proportion of cells with repressed URA3 (\%FOA $\left.{ }^{\mathrm{R}}\right)$ was assessed as the number of colonies on plates containing FOA (SC+FOA) divided by the number of colonies on non-selective plates. The difference in $\% \mathrm{FOA}^{\mathrm{R}}$ values is indicative of the difference in the levels of silencing at the analysed locus.

These analyses revealed that the destruction of the B1 element (TTT $\rightarrow$ ccT) in ARS1 moderately reduced the $\% \mathrm{FOA}^{\mathrm{R}}$ values suggesting that this $B 1$ element contributes to the overall gene silencing at telomeres (Fig. 4). Exactly the same mutation has also significantly reduced the replicator activity of ARS1 (Marahrens \& Stillman, 1992). Surprisingly, the destruction of the putative B1 element (ATT $\rightarrow$ ccT) of ARS319 had very little effect on telomeric silencing (Fig. 4) and only moderately reduced the replicator activity of ARS319 (Chang et al., 2008). These puzzling results suggest that ARS319 does not possess an ordinary B1 element. It is possible that B1 in ARS319 is offset from the customary position found in other ARSs. Alternatively, ARS319 has a broader B1 element that is not affected by the replacement of only two nucleotides.

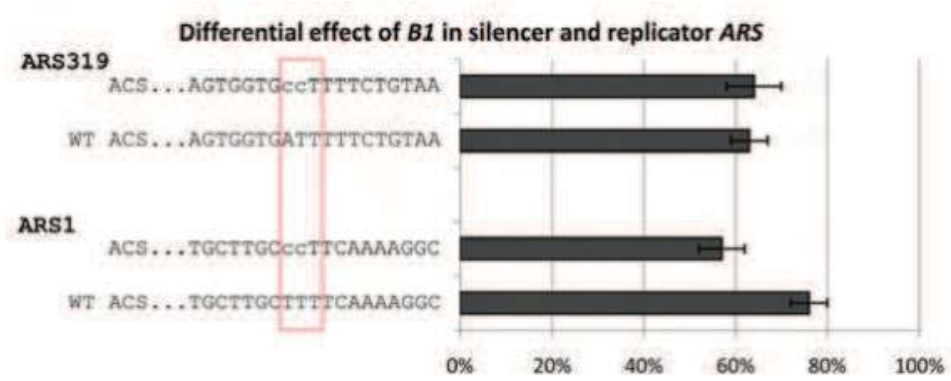

Fig. 4. Differential effect of $B 1$ in silencers and replicators - FOA sensitivity assays were performed to assess the level of silencing in mutant and wild type proto-silencer (ARS319) and replicator (ARS1) ARSs at the VII-L telomere in S.cerevisiae. Average \% FOA ${ }^{\mathrm{R}}$ with standard errors are shown. The hypothesized B1 WTW motif is indicated by the red rectangle. Site-directed mutations are indicated by lower-case letters above the wild type (WT) sequences. 


\subsection{Scanning mutations of the $B 1$ element in a silencer $A R S$ show little effect on its silencing and replicator activity}

ARS317 is a well-characterised core component of the HMRa- $E$ silencer. Similar to the protosilencer ARS319, mutations in the putative B1 element (TTA $\rightarrow$ Tcc) of ARS317 have little effect on its replicator activity (Chang et al., 2008). In order to assess the role of the ARS317-B1 element in gene silencing, we performed a two-nucleotide substitution scanning mutagenesis of the region encompassing its putative B1 (Fig. 5). All ACS-b1 mutants were cloned next to $U R A 3$ and inserted in the left telomere of chromosome VII as before. The level of repression of URA3 was assessed by the FOA sensitivity assay as described in the previous section. The results indicated that the destruction of the WTW motif (TTA $\rightarrow \mathrm{CCA}$ or TTA $\rightarrow$ TTC) did not reduce, but actually slightly increased the silencing of URA3 (Fig. 5). The only moderate decrease in silencing was observed in the construct 1.5, where a GC pair proximal to ACS was replaced with an AA (GC $\rightarrow$ aa). This result is somewhat surprising as $\mathrm{G} / \mathrm{C}$ bases do not conform to the general $\mathrm{A} / \mathrm{T}$ rich nature of this region. In conclusion, the canonical $B 1$ elements of both ARS317 and ARS319 seemed dispensable for silencer function (Fig. 4 and Fig. 5) and had little or no effect on the replicator activity of these ARSs (Chang et al., 2008).

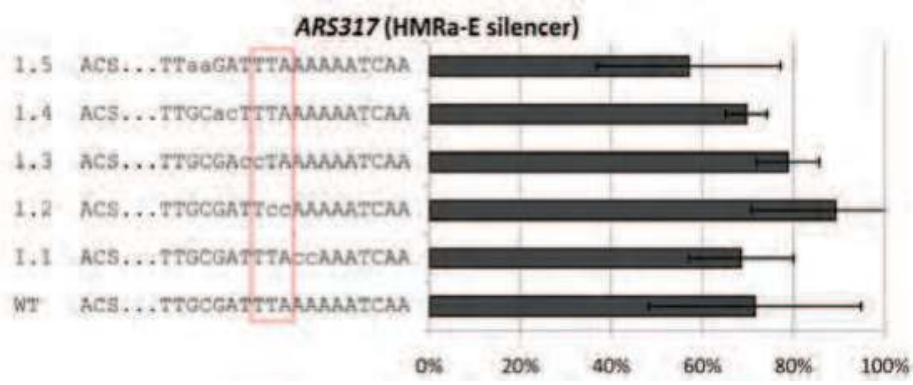

Fig. 5. FOA sensitivity of scanning mutations in B1 in ARS317 - FOA sensitivity assays were performed to assess the level of silencing in five mutants (1.1-1.5) and wild type (WT) ARS317 at the VII-L telomere in S.cerevisiae. Average \%FOAR with standard errors are shown. The hypothesized B1 WTW motif is indicated by the red rectangle. Site-directed substitution constructs are indicated by lower-case letters above the wild type (WT) sequence.

\subsection{Scanning mutations of the B1 element of a replicator $A R S$ affect both silencer and replicator function}

We conducted a similar scanning mutagenesis analysis of the $B 1$ element in one of the most active origins of DNA replication in the genome of S.cerevisiae, ARS305 (Huang \& Kowalski, 1996). Two-nucleotide substitutions were introduced at the positions shown in Fig. 6 and the mutant ACS- $b 1$ constructs were attached to URA3. These reporter cassettes were inserted in the left telomere of chromosome VII and analysed for the levels of URA3 repression. The analysis showed that, similar to ARS1, the destruction of B1 in ARS305 reduced the levels of gene silencing at the VII-L telomere (Fig. 6). In Fig. 6, it is interesting to note that the 1.1 construct (substitution of one base from the WTW motif and one adjacent base) did not reduce the silencing ability while the 1.2 construct (substitution of two bases in the WTW motif) caused silencing ability to decrease by approximately $15 \%$. Also, it is curious that mutations in the WTW flanking sequences (constructs 1.3 and 1.5) showed the greatest 


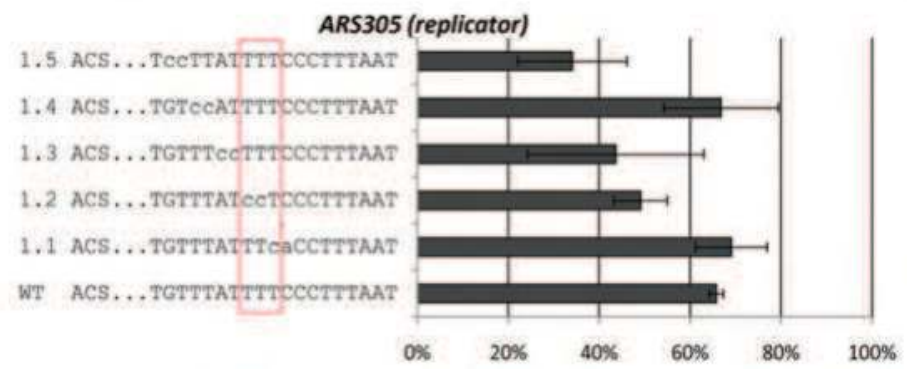

Fig. 6. FOA sensitivity of scanning mutations in B1 in ARS305 - FOA sensitivity assays were performed to assess the level of silencing in five scanning mutants (1.1-1.5) and wild type (WT) ARS305 at the VII-L telomere in S.cerevisiae. Average \% FOA ${ }^{\mathrm{R}}$ with standard errors are shown. The hypothesized B1 WTW motif is indicated by the red rectangle. Site-directed substitution constructs are indicated by lower-case letters above the wild type (WT) sequence.

reductions in silencing ( $30 \%$ and $20 \%$ reductions of $\% \mathrm{FOA}^{\mathrm{R}}$ respectively). Very similar mutations have been shown to affect the activity of ARS305 as a replicator (Huang \& Kowalski, 1996). So, the two replicator ARSs we have analysed possess a well preserved B1 element, which functions in both origin firing and in epigenetic silencing. Also, scanning substitutions of the ARS305 B1 element revealed that mutations in the B1 flanking sequences have significant effects on gene silencing as well. In contrast, the two silencer ARSs were unaffected by any of the two-nucleotide substitutions in the B1 region.

\subsection{Is there any substantial difference to $B 1$ in replicators and silencers?}

The subtle differences in the activities of $B 1$ elements in select replicator and silencer $A R S s$ prompted us to perform extensive sequence alignments of multiple $A R S$ s according to their function and/or location in the genome. ARSs were grouped as replicators (ARSs that are located away from the telomeres and the silencer loci, which confer autonomous replication when moved to a plasmid), silencers (ARSs from the HM loci and the $r D N A$ locus) and ARSs within $5 \mathrm{~kb}$ of the telomeres. We note that the latter category contains ARSs that are imbedded in the repetitive Core $X$ and $Y^{\prime}$ subtelomeric elements (Chan \& Tye, 1983; Walmsley et al., 1984) and that they share higher homology in the sequences outside the ACS. All sequences were imported in WebLogo (www.weblogo.berkeley.edu) and analysed for similarities (Fig. 7).

These analyses confirmed the higher sequence conservation in the vicinity of replicator B1 elements that was reported earlier (Chang et al., 2008). Nevertheless, telomeric proto-silencer and silencer ARSs seem to contain broader WTTTTT and WTTT consensus sequences, respectively, as compared to the WTW consensus of the replicators. These slight variations corroborate the differences observed in the scanning mutation analyses of ARS305 (replicator) and ARS317 (silencer). It is quite possible that the broader A/T rich stretch in B1 of the silencer $A R S$ s contribute to the lower effect of the two-nucleotide substitutions in ARS317 as compared to ARS305 (Figs. 5 and 6). However, we need to stress that both the effects in the silencing assays and the difference in the $B 1$ sequence are subtle and do not really reveal a major feature that can distinguish between the two types of ARS. 


\subsection{The orientation of ACS-B1 determines the levels of telomeric gene silencing}

Previous studies have indicated that the orientation of the HMR a and HML $\alpha$ silencers impose directional repression of genes (Zou et al., 2006a; Zou et al., 2006b). In particular, the $H M L-I$ and the HMR-E silencers were found to more efficiently repress URA3 reporters if oriented B1-ACS-Rap1-Abf1-URA3 (Fig. 2) (Zou et al., 2006b). These effects were linked to the ability of ACS (and supposedly ORC) to robustly position a nucleosome towards the Abf1 side of the silencer (Zou et al., 2006a). Towards the B1 side of ACS there is no stably positioned nucleosome and the silencing of $U R A 3$ is significantly weaker. Interestingly, the replicator ARS1 has a stably positioned nucleosome at both the B1 and the Abf1 sides of ACS (Lipford \& Bell, 2001; Zou et al., 2006a).

\section{A) Replicator ARSs}

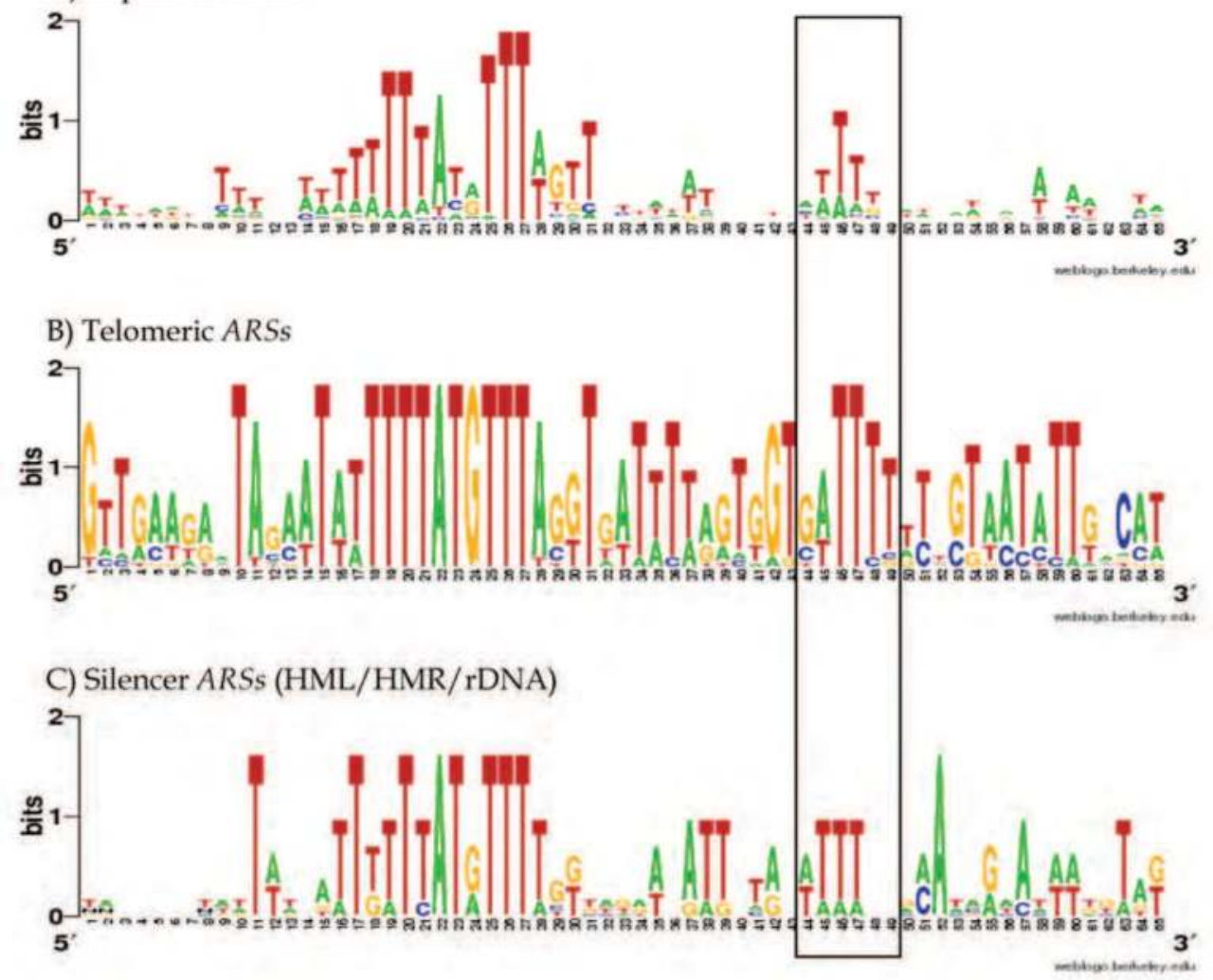

Fig. 7. Sequence alignments of replicator, proto-silencer and silencer ARSs - WebLogo alignments were performed using $A R S$ sequences from $S G D$ (www.yeastgenome.org). Sequences were aligned along the ACS (5'-WTTTAYRTTTW-3') and include 17 bp upstream and $37 \mathrm{bp}$ downstream of $A C S$. The $B 1$ element is indicated by the black rectangle. ARSs were chosen based on: A) non-telomeric location with known replicator activity (25 ARSs included); B) proximity to telomere (within $5 \mathrm{~kb}$ ) and confirmed autonomous replication on a mini-chromosome (13 ARSs included); C) non-telomeric location and confirmed silencer or proto-silencer activity (HML/HMR/rDNA, 6 ARSs included). 
It is not entirely clear what determines the directional effects of the $H M L-I$ and $H M R-E$ silencers. For example, do Abf1p and Rap1p (and presumably other proto-silencers) facilitate the rigorous control of the nucleosome positions or does ACS-B1/ORC work independently? In this line of thought, many origins (such as ARS305, ARS307, ARS605) do not contain Abf1p or Rap1p binding sites, while in others (ARS1, ARS319) B1 and the Abf1p binding site reside on the same side of ACS (Marahrens \& Stillman, 1992; Rao et al., 1994; Huang \& Kowalski, 1996; Rehman et al., 2009). Invariably, all these ARSs improve gene silencing when inserted at the telomere ((Rehman et al., 2009) this article). We decided to test if these $A R S$ s also display directional silencing.

Initial experiments were conducted using ARS605 and ARS319. As mentioned earlier, ARS605 has no apparent Abf1p binding site, while in ARS319 both B1 and the Abf1p binding sites are at the same side of $A C S$. We have cloned these origins in both orientations relative to URA3 and the telomere to produce the URA3-ACS605-B1-tel, URA3-B1-ACS605tel, URA3-ACS319-B1-tel and URA3-B1-ACS319-tel constructs. These constructs were inserted in the left telomere of chromosome $V I I$ and the levels of URA3 repression were assessed as before (Fig. 8).

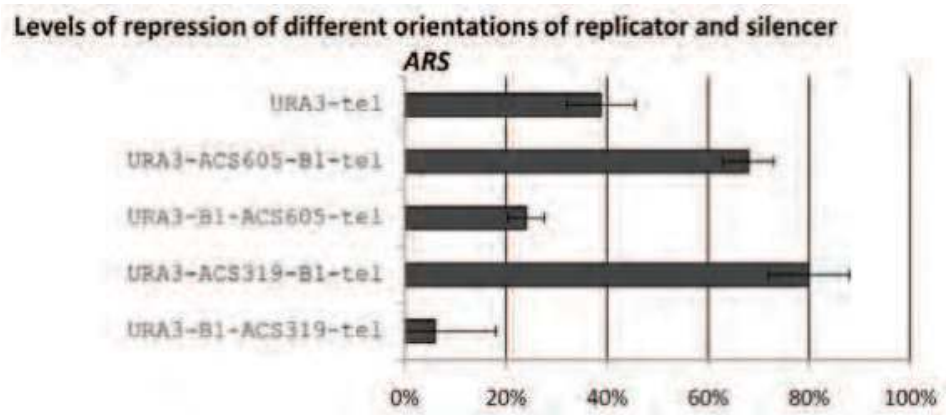

Fig. 8. Effect of the orientation of $A C S-B 1$ on telomeric silencing - FOA sensitivity assays were performed to assess the level of silencing in two ACS-B1 orientations for ARS605 and ARS319 at the VII-L telomere in S.cerevisiae. URA3-tel acted as a control showing level of silencing when no proto-silencer is present. Average $\% \mathrm{FOA}^{\mathrm{R}}$ with standard errors are shown.

Our results indicate that both ARS319 and ARS605 boost the repression of URA3 in the URA3-ACS-B1-tel orientation. These results are in tune with earlier observations (Zou et al., 2006a; Zou et al., 2006b). Very interestingly, in the opposite direction these ARSs markedly reduced the repression of URA3 (Fig. 8). So, similar to HML-I (ARS302) and HMR-E (ARS317), ARS605 and ARS319 display directional silencing, but also act as anti-silencers in the opposite direction. Assuming that a similar robustly positioned nucleosome next to ACS determines the direction of silencing of ARS605 and ARS319, we argue that the lack of a stable nucleosome at the $B 1$ side of these $A C S$ s can serve as an insulator against the spreading of SIR proteins from the telomere and dampen silencing. We also suggest that other ARSs including ARS302 and ARS317 will have a similar insulating activity. Together, our results indicate that the orientation of ORC towards a nearby silencing domain (such as the telomere or the HM loci) has a significant impact on the strength of silencing. 


\section{Discussion}

\subsection{Role of the $B 1$ element in ARS duality}

The central topic of the presented studies is the enigmatic dual function of ARSs as replicators and as silencers. Because earlier studies have shown distinct affinity of ORC to $A R S$ s in silencers and replicators and because $B 1$ has been proposed to affect ORC affinity (Palacios DeBeer et al., 2003), we have focused on the role of this element on gene silencing at the telomeres. We have compared our results to similar analyses on the role of B1 in origin activity.

We have found that mutations in the B1 elements of replicator ARSs reduce their activity in telomeric silencing (Figs. 4 and 6). Similar mutations have also reduced the replicator activity of these ARSs (Chang et al., 2008; Marahrens \& Stillman, 1992). So, replicator ARSs seem to have a well defined $B 1$ that is important, but not required, for both replication and silencing. However, mutations in the B1 elements of silencer ARSs seem not to affect silencing (Figs. 4-5). Similarly, mutations in the $B 1$ of these $A R S s$ have a lesser effect on replication activity than the effects seen in replicator ARSs (Chang et al., 2008; Marahrens \& Stillman, 1992). This leads us to the hypothesis that silencer $A R S s$ have a special type of $B 1$ element. In this line of thought, we have also noticed that mutations in the sequences flanking the WTW motif in replicator ARSs affect silencing to a greater extent than silencer ARSs (Fig. 6). This observation suggests that the sequences flanking WTW are more important for silencing than for replication and argue in favour of a broader B1 element in silencer ARSs.

We propose that the subtle functional differences between silencer and replicator ARSs is due to the broader $B 1$ consensus sequence in silencers. We suggest that silencer $A R S s$ contain more A/T base pairs around the WTW motif. Support for this hypothesis was provided by the alignment of different types of $A R S$ s. In Fig. 7, we introduced the notion of wider B1 elements, where a consensus of WTTTTT was found for proto-silencer ARSs and WTTT was found for silencer ARSs. Replicator ARSs showed only the previously described WTW motif (Chang et al., 2008). It is possible that ARSs with broad B1 elements would be more accommodating to mutations within the consensus because the adjacent bases would still resemble a WTW motif. The adjacent WTW sites may be able to act as alternative sites for the attachment of ORC (Fig. 9). It is also possible that these ARSs possess additional B1 elements that render the mutations in WTW insignificant.

Earlier structure-function analyses of the association of ORC to ARS1 (Rao \& Stillman, 1995) have indicated that ORC binds to both ACS and B1 (Fig. 9). Through cross-linking studies, a third minor position of ORC contact with DNA (depicted by "nnn" in Fig. 9) has also been revealed in-between ACS and B1 (Rao \& Stillman, 1995). This third site has never been shown to influence the activity of $A R S 1$, but could be important for fine conformational variations in ORC. On the other hand, the small effects of $B 1$ in replicator and silencer assays stress its auxiliary nature. Whereas ACS is required for interaction with ORC, the precise roles of $B 1$ and the " $n n n$ " sequences remain elusive. It is possible that these auxiliary sequences are adaptor elements, which modulate alternative structures of ORC (Fig. 9).

We can imagine that some of these alternative structures would not expose Orc1p, reducing its interaction with Sir1p, and thus promoting replicator activity instead of silencing. Other conformations of ORC, which expose Orc1p, would stimulate its interaction with Sir1p and increase the $A R S^{\prime}$ 's silencer activity. The broader B1 element present in proto-silencer and silencer $A R S$ s may allow ORC more binding flexibility than the narrower WTW in replicator ARSs. This increased flexibility may result in greater variations in ORC conformation, thus 
providing more opportunities for Orc1p to be exposed. A broader B1 can also provide additional sites for the attachment of ORC and in turn increase the affinity of ORC to ACS-B1. Such a scenario can explain the previous observations on the link between ORC affinity and stronger silencing. In particular, it has been discovered that strong ORC-DNA interaction at HMRa increased heterochromatin formation and decreased and delayed the initiation of DNA replication. Conversely, weak ORC-DNA interaction caused earlier and increased replication initiation and decreased the formation of heterochromatin (Palacios DeBeer et al., 2003).

Very importantly, alterations in $B 1$ by no means eliminate the dual nature of $A R S$. Whereas broad B1 elements seem to prevail in silencer and proto-silencer ARSs (Fig. 7), many replicator $B 1$ elements reside in an $\mathrm{A} / \mathrm{T}$ rich environment as well. This environment can also supply alternative sites for ORC binding. In summary, the B1 elements seem to unveil a minor difference between replicators and silencers, but this difference is not strong enough on its own to determine the function of an ARS.

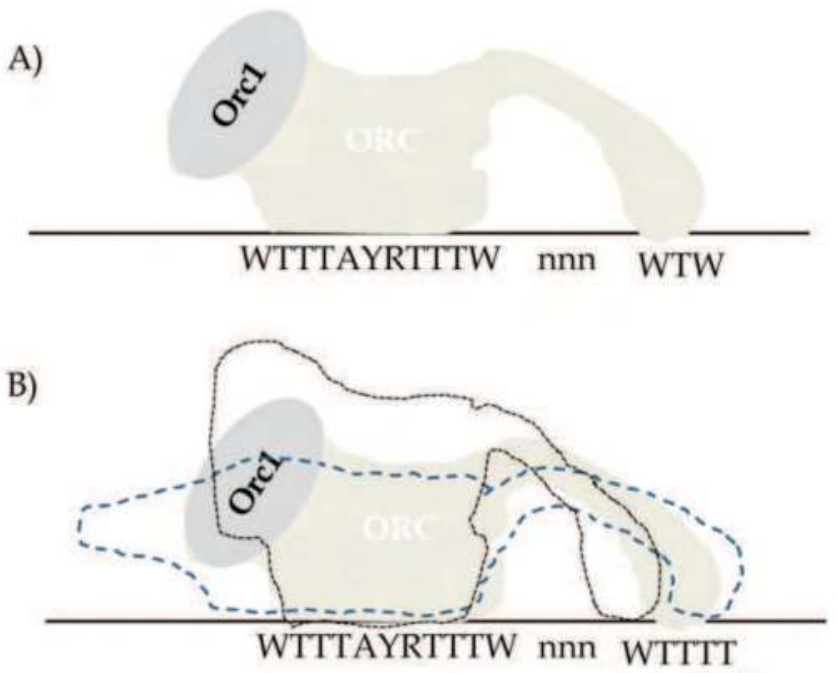

Fig. 9. ORC conformations as dictated by the ACS-B1 elements - ORC binds the bipartite $A C S-B 1$ site. The ACS (WTTTAYRTTTW) is essential for ORC binding while B1 (WTW) and a third minor position of interaction (nnn) are auxiliary. A) Depiction of the conformation of ORC when bound to an ARS with a distinct WTW B1 element. B) Depiction of the flexibility of ORC to adjust its conformation when the B1 consensus is broader (WTTTT), showing that it is possible that some conformations may hide or further expose the Orc1p subunit.

\subsection{Flexibility of ORC and the role of chromatin in $A R S$ duality}

The orientation of $A C S-B 1$ towards a potent silencer seems to be more important than the nature of B1. In support, we (Fig. 8) and others (Zou et al., 2006a; Zou et al., 2006b) have found that the orientation of $A R S$ s towards a powerful silencer such as the telomere or the $H M$ loci can significantly contribute to gene silencing. These effects suggest that ORC is highly flexible since a simple switch of direction contributes so significantly to silencing and anti-silencing. An interesting experiment would be to test how the replicator activity of $A R S s$ is affected based on its orientation towards a silencer. 
It is well known that the origin activity of an ARS is governed by chromatin structure (Weinreich et al., 2004). As mentioned previously, both replicator and silencer ARSs act as functional origins of replication when placed on plasmids (Chan \& Tye, 1980). Similarly, both replicator and silencer $A R S$ s act as functional silencers and proto-silencers in the HM loci and at the subtelomeres, respectively (Casey et al., 2008; McNally \& Rine, 1991; Weinreich et al., 2004). Since an ARS's location within the genome determines its function regardless of its original silencer/replicator classification, it appears that the sequence of the $A R S s$ is of lesser importance than the genomic context. Hence, it seems that the communication of ORC with heterochromatin is more important than the way ORC interacts with $A C S-B 1$. Again, ORC is posing as a highly flexible complex, this time in regards to its interaction with chromatin.

Our ideas that the flexibility of ORC can be influenced by chromatin feed some thought on how origins are chosen as per the Jesuit model. In metazoans, the positions of potential origins and origins that actually fire will vary depending on the epigenetic state of the genome. It makes sense not to fire origins that will disturb heterochromatin. One of the ways to ensure that this does not happen is to force the key regulatory factor for origin activation (this being ORC) to function in a different mode. This way, though ORC interacts with all of the potential origins, it will recruit replication machinery only if it is in a euchromatic region. If ORC is bound to DNA that lies within a heterochromatic region, the heterochromatin dictates that ORC will only recruit silencing machinery and maintain the heterochromatic state instead of stimulating replication.

\section{Conclusion}

Despite our extensive efforts, we have not identified a clear and strong distinctive feature for the replicator or the silencer ARSs. This brings us back to the idea that ARS, together with the associated ORC and other DNA replication factors, is a flexible bi-functional module that can be remoulded depending on the chromosomal context and perhaps by additional factors such as the need of the cell to divide or to modulate its gene expression. In other eukaryotes we see no readily identifiable consensus sequence in origins of DNA replication (Mechali, 2010). Even so, strong links of ORC to gene silencing and heterochromatin have been identified by many studies in Schizosaccharomyces pombe and in higher eukaryotes (Pak et al., 1997; Auth et al., 2006; Deng et al., 2007; Stuermer et al., 2007; Kato et al., 2008; Deng et al., 2009; Prasanth et al., 2010). Perhaps ORC has evolved to lose its stringent sequence requirements for binding to DNA, but the origin-ORC module has maintained its flexibility and the ability to accommodate varying conformations.

It is somewhat anecdotal that while looking for updates on the Jesuit model for the firing of metazoan origins (DePamphilis, 1993) we came across this citation dealing with the duality of the universe: "...Light and Darkness, Life and Death, Right and Left.... are inseparable.... For this reason each one will dissolve into its earliest Origin..." (The Gospel of Philip, New Testament Apocrypha). It seems that duality has been encripted in the earliest origin and then preserved through evolution.

\section{Acknowldegements}

The studies in this article have been supported by grants to K.Yankulov from NSERC. D. Jeffery has been supported by studentships from OGSST and from NSERC. P. ChisamoreRobert is supported through stipend from the University of Guelph and NSERC. 


\section{References}

Abraham, J., Feldman, J., Nasmyth, K.A., Strathern, J.N., Klar, A.J., Broach, J.R., \& Hicks, J.B. (1983). Sites required for position-effect regulation of mating-type information in yeast. Cold Spring Harb Symp Quant Biol 47 Pt 2, 989-998.

Auth, T., Kunkel, E., \& Grummt, F. (2006). Interaction between HP1alpha and replication proteins in mammalian cells. Exp Cell Res 312, 3349-3359.

Axelrod, A., \& Rine, J. (1991). A role for CDC7 in repression of transcription at the silent mating-type locus HMR in Saccharomyces cerevisiae. Mol Cell Biol 11, 1080-1091.

Blow, J.J., \& Dutta, A. (2005). Preventing re-replication of chromosomal DNA. Nat Rev Mol Cell Biol 6, 476-486.

Broach, J.R., Li, Y.Y., Feldman, J., Jayaram, M., Abraham, J., Nasmyth, K.A., \& Hicks, J.B. (1983). Localization and sequence analysis of yeast origins of DNA replication. Cold Spring Harb Symp Quant Biol 47 Pt 2, 1165-1173.

Casey, L., Patterson, E.E., Muller, U., \& Fox, C.A. (2008). Conversion of a replication origin to a silencer through a pathway shared by a Forkhead transcription factor and an $\mathrm{S}$ phase cyclin. Mol Biol Cell 19, 608-622.

Chan, C.S., \& Tye, B.K. (1980). Autonomously replicating sequences in Saccharomyces cerevisiae. Proc Natl Acad Sci U S A 77, 6329-6333.

Chan, C.S., \& Tye, B.K. (1983). Organization of DNA sequences and replication origins at yeast telomeres. Cell 33, 563-573.

Chang, F., Theis, J.F., Miller, J., Nieduszynski, C.A., Newlon, C.S., \& Weinreich, M. (2008). Analysis of chromosome III replicators reveals an unusual structure for the ARS318 silencer origin and a conserved WTW sequence within the origin recognition complex binding site. Mol Cell Biol 28, 5071-5081.

Chien, C.T., Buck, S., Sternglanz, R., \& Shore, D. (1993). Targeting of SIR1 protein establishes transcriptional silencing at HM loci and telomeres in yeast. Cell 75, 531-541.

Deng, Z., Dheekollu, J., Broccoli, D., Dutta, A., \& Lieberman, P.M. (2007). The origin recognition complex localizes to telomere repeats and prevents telomere-circle formation. Curr Biol 17, 1989-1995.

Deng, Z., Norseen, J., Wiedmer, A., Riethman, H., \& Lieberman, P.M. (2009). TERRA RNA binding to TRF2 facilitates heterochromatin formation and ORC recruitment at telomeres. Mol Cell 35, 403-413.

DePamphilis, M.L. (1993). Eukaryotic DNA replication: anatomy of an origin. Annu Rev Biochem 62, 29-63.

Doyon, Y., Cayrou, C., Ullah, M., Landry, A.J., Cote, V., Selleck, W., Lane, W.S., Tan, S., Yang, X.J., \& Cote, J. (2006). ING tumor suppressor proteins are critical regulators of chromatin acetylation required for genome expression and perpetuation. Mol Cell 21, 51-64.

Ehrenhofer-Murray, A.E., Kamakaka, R.T., \& Rine, J. (1999). A role for the replication proteins PCNA, RF-C, polymerase epsilon and Cdc45 in transcriptional silencing in Saccharomyces cerevisiae. Genetics 153, 1171-1182.

Ehrentraut, S., Weber, J.M., Dybowski, J.N., Hoffmann, D., \& Ehrenhofer-Murray, A.E. (2010). Rpd3-dependent boundary formation at telomeres by removal of Sir2 substrate. Proc Natl Acad Sci U S A 107, 5522-5527.

Espinosa, M.C., Rehman, M.A., Chisamore-Robert, P., Jeffery, D., \& Yankulov, K. (2010). GCN5 is a positive regulator of origins of DNA replication in Saccharomyces cerevisiae. PLoS One 5, e8964. 
Field, Y., Kaplan, N., Fondufe-Mittendorf, Y., Moore, I.K., Sharon, E., Lubling, Y., Widom, J., \& Segal, E. (2008). Distinct modes of regulation by chromatin encoded through nucleosome positioning signals. PLoS Comput Biol 4, e1000216.

Fourel, G., Lebrun, E., \& Gilson, E. (2002). Protosilencers as building blocks for heterochromatin. Bioessays 24, 828-835.

Fourel, G., Magdinier, F., \& Gilson, E. (2004). Insulator dynamics and the setting of chromatin domains. Bioessays 26, 523-532.

Fourel, G., Revardel, E., Koering, C.E., \& Gilson, E. (1999). Cohabitation of insulators and silencing elements in yeast subtelomeric regions. EMBO J 18, 2522-2537.

Huang, R.Y., \& Kowalski, D. (1996). Multiple DNA elements in ARS305 determine replication origin activity in a yeast chromosome. Nucleic Acids Res 24, 816-823.

Kato, H., Matsunaga, F., Miyazaki, S., Yin, L., D'Urso, G., Tanaka, K., \& Murakami, Y. (2008). Schizosaccharomyces pombe Orc5 plays multiple roles in the maintenance of genome stability throughout the cell cycle. Cell Cycle 7, 1085-1096.

Kearsey, S. (1983). Analysis of sequences conferring autonomous replication in baker's yeast. EMBO J 2, 1571-1575.

Labib, K. (2010). How do Cdc7 and cyclin-dependent kinases trigger the initiation of chromosome replication in eukaryotic cells? Genes Dev 24, 1208-1219.

Lafon, A., Chang, C.S., Scott, E.M., Jacobson, S.J., \& Pillus, L. (2007). MYST opportunities for growth control: yeast genes illuminate human cancer gene functions. Oncogene 26, 5373-5384.

Lin, S., \& Kowalski, D. (1997). Functional equivalency and diversity of cis-acting elements among yeast replication origins. Mol Cell Biol 17, 5473-5484.

Lipford, J.R., \& Bell, S.P. (2001). Nucleosomes positioned by ORC facilitate the initiation of DNA replication. Mol Cell 7, 21-30.

Marahrens, Y., \& Stillman, B. (1992). A yeast chromosomal origin of DNA replication defined by multiple functional elements. Science $255,817-823$.

McNally, F.J., \& Rine, J. (1991). A synthetic silencer mediates SIR-dependent functions in Saccharomyces cerevisiae. Mol Cell Biol 11, 5648-5659.

Mechali, M. (2010). Eukaryotic DNA replication origins: many choices for appropriate answers. Nat Rev Mol Cell Biol 11, 728-738.

Nieduszynski, C.A., Knox, Y., \& Donaldson A.D. (2006). Genome-wide identification of replication origins in yeast by comparative genomics. Genes Dev. Vol. 20, 14, pp. (1874-1879)

Pak, D.T., Pflumm, M., Chesnokov, I., Huang, D.W., Kellum, R., Marr, J., Romanowski, P., \& Botchan, M.R. (1997). Association of the origin recognition complex with heterochromatin and HP1 in higher eukaryotes. Cell 91, 311-323.

Palacios DeBeer, M.A., Muller, U., \& Fox, C.A. (2003). Differential DNA affinity specifies roles for the origin recognition complex in budding yeast heterochromatin. Genes Dev 17, 1817-1822.

Power, P., Jeffery, D., Rehman, M.A., Chatarji, A, \& K. Yankulov. (2011). Sub-Telomeric core $X$ and $Y^{\prime}$ Elements in S.cerevisiae Suppress Extreme Variations in Gene Silencing PLoS One 6(3), e17523.

Prasanth, S.G., Shen, Z., Prasanth, K.V., \& Stillman, B. (2010). Human origin recognition complex is essential for HP1 binding to chromatin and heterochromatin organization. Proc Natl Acad Sci U S A 107, 15093-15098. 
Pryde, F.E., \& Louis, E.J. (1999). Limitations of silencing at native yeast telomeres. EMBO J $18,2538-2550$.

Raghuraman, M.K., Winzeler, E.A., Collingwood, D., Hunt, S., Wodicka, L., Conway, A., Lockhart, D.J., Davis, R.W., Brewer, B.J., \& Fangman, W.L. (2001). Replication dynamics of the yeast genome. Science 294, 115-121.

Rao, H., Marahrens, Y., \& Stillman, B. (1994). Functional conservation of multiple elements in yeast chromosomal replicators. Mol Cell Biol 14, 7643-7651.

Rao, H., \& Stillman, B. (1995). The origin recognition complex interacts with a bipartite DNA binding site within yeast replicators. Proc Natl Acad Sci U S A 92, 2224-2228.

Rehman, M.A., Fourel, G., Mathews, A., Ramdin, D., Espinosa, M., Gilson, E., \& Yankulov, K. (2006). Differential requirement of DNA replication factors for subtelomeric ARS consensus sequence protosilencers in Saccharomyces cerevisiae. Genetics 174, 18011810.

Rehman, M.A., Wang, D., Fourel, G., Gilson, E., \& Yankulov, K. (2009). Subtelomeric ACScontaining proto-silencers act as antisilencers in replication factors mutants in Saccharomyces cerevisiae. Mol Biol Cell 20, 631-641.

Rehman, M.A., \& Yankulov, K. (2009). The dual role of autonomously replicating sequences as origins of replication and as silencers. Curr Genet 55, 357-363.

Rusche, L.N., Kirchmaier, A.L., \& Rine, J. (2003). The establishment, inheritance, and function of silenced chromatin in Saccharomyces cerevisiae. Annu Rev Biochem 72, 481-516.

Sheu, Y.J., \& Stillman, B. (2010). The Dbf4-Cdc7 kinase promotes S phase by alleviating an inhibitory activity in Mcm4. Nature 463, 113-117.

Shore, D., \& Nasmyth, K. (1987). Purification and cloning of a DNA binding protein from yeast that binds to both silencer and activator elements. Cell 51, 721-732.

Shore, D., Stillman, D.J., Brand, A.H., \& Nasmyth, K.A. (1987). Identification of silencer binding proteins from yeast: possible roles in SIR control and DNA replication. EMBO J 6, 461-467.

Stinchcomb, D.T., Struhl, K., \& Davis, R.W. (1979). Isolation and characterisation of a yeast chromosomal replicator. Nature 282, 39-43.

Stuermer, A., Hoehn, K., Faul, T., Auth, T., Brand, N., Kneissl, M., Putter, V., \& Grummt, F. (2007). Mouse pre-replicative complex proteins colocalise and interact with the centrosome. Eur J Cell Biol 86, 37-50.

Walmsley, R.W., Chan, C.S., Tye, B.K., \& Petes, T.D. (1984). Unusual DNA sequences associated with the ends of yeast chromosomes. Nature 310, 157-160.

Weinreich, M., Palacios DeBeer, M.A., \& Fox, C.A. (2004). The activities of eukaryotic replication origins in chromatin. Biochim Biophys Acta 1677, 142-157.

Wyrick, J.J., Aparicio, J.G., Chen, T., Barnett, J.D., Jennings, E.G., Young, R.A., Bell, S.P., \& Aparicio, O.M. (2001). Genome-wide distribution of ORC and MCM proteins in S. cerevisiae: high-resolution mapping of replication origins. Science 294, 2357-2360.

Zou, Y., Yu, Q., \& Bi, X. (2006a). Asymmetric positioning of nucleosomes and directional establishment of transcriptionally silent chromatin by Saccharomyces cerevisiae silencers. Mol Cell Biol 26, 7806-7819.

Zou, Y., Yu, Q., Chiu, Y.H., \& Bi, X. (2006b). Position effect on the directionality of silencer function in Saccharomyces cerevisiae. Genetics 174, 203-213. 


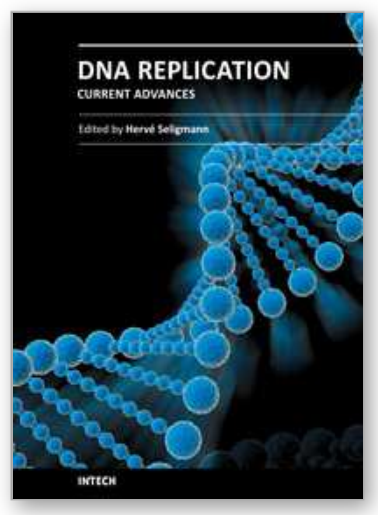

\section{DNA Replication-Current Advances}

Edited by Dr Herve Seligmann

ISBN 978-953-307-593-8

Hard cover, 694 pages

Publisher InTech

Published online 01, August, 2011

Published in print edition August, 2011

The study of DNA advanced human knowledge in a way comparable to the major theories in physics, surpassed only by discoveries such as fire or the number zero. However, it also created conceptual shortcuts, beliefs and misunderstandings that obscure the natural phenomena, hindering its better understanding. The deep conviction that no human knowledge is perfect, but only perfectible, should function as a fair safeguard against scientific dogmatism and enable open discussion. With this aim, this book will offer to its readers 30 chapters on current trends in the field of DNA replication. As several contributions in this book show, the study of DNA will continue for a while to be a leading front of scientific activities.

\section{How to reference}

In order to correctly reference this scholarly work, feel free to copy and paste the following:

Patricia Chisamore-Robert, Daniel Jeffery and Krassimir Yankulov (2011). The Silencing Face of DNA Replication: Gene Repression Mediated by DNA Replication Factors, DNA Replication-Current Advances, Dr Herve Seligmann (Ed.), ISBN: 978-953-307-593-8, InTech, Available from:

http://www.intechopen.com/books/dna-replication-current-advances/the-silencing-face-of-dna-replicationgene-repression-mediated-by-dna-replication-factors

\section{INTECH}

open science | open minds

\section{InTech Europe}

University Campus STeP Ri

Slavka Krautzeka 83/A

51000 Rijeka, Croatia

Phone: +385 (51) 770447

Fax: +385 (51) 686166

www.intechopen.com

\section{InTech China}

Unit 405, Office Block, Hotel Equatorial Shanghai

No.65, Yan An Road (West), Shanghai, 200040, China 中国上海市延安西路65号上海国际贵都大饭店办公楼 405 单元

Phone: +86-21-62489820

Fax: $+86-21-62489821$ 
(C) 2011 The Author(s). Licensee IntechOpen. This chapter is distributed under the terms of the Creative Commons Attribution-NonCommercialShareAlike-3.0 License, which permits use, distribution and reproduction for non-commercial purposes, provided the original is properly cited and derivative works building on this content are distributed under the same license. 